\title{
ZETA AND NORMAL ZETA FUNCTIONS FOR A SUBCLASS OF SPACE GROUPS
}

\author{
HERMINA ALAJBEGOVIĆ AND MUHAREM AVDISPAHIĆ
}

\begin{abstract}
We calculate zeta and normal zeta functions of space groups with the point group isomorphic to the cyclic group of order 2. The obtained results are applied to determine the number of subgroups, resp. normal subgroups, of a given index for each of these groups.
\end{abstract}

Keywords: zeta functions of groups, crystallographic groups

Mathematics Subject Classification (2010) 11M41, $20 \mathrm{H} 15$

\section{Introduction}

The concepts of the zeta and normal zeta function of a group were introduced by Smith, Segal and Grunewald in [4] and [7]. The zeta function of a group $G$ is defined as $\zeta_{G}(s)=\sum_{n \in \mathbb{N}} a_{n}(G) n^{-s}$, where $a_{n}(G)$ denotes the number of subgroups of index $n$ in $G$. The normal zeta function of a group $G$ is given by $\zeta_{G}^{\triangleleft}(s)=\sum_{n \in \mathbb{N}} c_{n}(G) n^{-s}$, where $c_{n}(G)$ is the number of normal subgroups of index $n$ in $G$. These functions provide a useful tool for studying the relationship between the asymptotic behavior of the sequences $a_{n}(G)$, resp. $c_{n}(G)$, and the structure of $G$.

If a group $G$ has a polynomial subgroup growth, i.e. if $a_{n}(G) \leq n^{k}$ for some $k$ and for all $n \in N$, then $\zeta_{G}(s)$ has a non - trivial domain of convergence. In particular, if $G$ is residually finite nilpotent group then $a_{n}(G)$ grows polynomially and $\zeta_{G}(s)=\sum_{n \in \mathbb{N}} a_{n}(G) n^{-s}$ satisfies an Euler product formula $\zeta_{G}(s)=\prod_{p \in P} \zeta_{G, p}(s)$, where $\zeta_{G, p}(s)$ counts only subgroups of $p$ - power index and $P$ denotes the set of all primes.

M. P. F. du Sautoy, J. J. McDermott and G. C. Smith [2] proved the following theorem.

Theorem 1.1. Let $G$ be a finite extension of a free abelian group of finite rank. Then $\zeta_{G}(s)$ and $\zeta_{G}^{\triangleleft}(s)$ can be extended to meromorphic functions on the whole complex plane.

Lubotzky and du Sautoy [1] established a functional equation $\left.\zeta_{G, p}(s)\right|_{p \rightarrow p^{-1}}=(-1)^{n} p^{a s+b} \zeta_{G, p}(s)$ satisfied by the local factors of the zeta function of a group for some torsion-free nilpotent groups and appropriate $a, b, n \in \mathbb{N}$. Here $p \rightarrow p^{-1}$ denotes a formal inversion of the local parameter $p$. In this regard, see also [8], [9].

There are relatively few explicit expressions known for zeta functions of groups. John J. McDermott calculated the zeta functions of the seventeen plane crystallographic groups in [6].

A space group represents a description of the symmetry of a crystal. A crystallographic group $G$ contains a translation subgroup $T$ which consists of all elements of the group corresponding to 
translations of the pattern involved. The factor group $G / T$ is known as the point group of $G$ and is denoted by $P$. Group $G$ is a finite extension of $T$ by $P$, since $P$ is finite.

In this paper, we calculate zeta and normal zeta functions of space groups with the point group isomorphic to the cyclic group of order 2 . There are eight such groups: $P \overline{1}, P 2, P 2_{1}, C 2, P m, P c, C m$ and $C c$ [5]. After stating results in Section 2, we provide the application of these results to compute the number of subgroups of a given index for each group in Section 3 and Section 4 . We describe the method of proof in Section 5 and provide full details in case of the group C2, as a sample.

\section{Results}

For a sake of bravity, the following notation for translates of the Riemann zeta function is used in the sequel:

$\zeta_{k}(s)=\zeta(s-k)$, i.e., $\zeta_{2}(s)=\zeta(s-2)$.

Theorem 2.1. Zeta functions of space groups with the point group isomorphic to the cyclic group of order 2 read as follows

$$
\begin{aligned}
& \zeta_{P \overline{1}}(s)=\zeta_{1}(s) \zeta_{2}(s) \zeta_{3}(s)+2^{-s} \zeta(s) \zeta_{1}(s) \zeta_{2}(s) \\
& \zeta_{P 2}(s)=\left(1+2^{-s+3}\right) \zeta(s) \zeta_{1}(s) \zeta_{2}(s) \\
& \zeta_{P 21}(s)=\zeta(s) \zeta_{1}(s) \zeta_{2}(s) \\
& \zeta_{C 2}(s)=\left(1+2^{-2 s+3}\right) \zeta(s) \zeta_{1}(s) \zeta_{2}(s) \\
& \zeta_{P m}(s)=\left(1+9 \cdot 2^{-s}+6 \cdot 2^{-2 s}\right) \zeta(s) \zeta_{1}(s) \zeta_{1}(s)+2^{-s} \zeta(s) \zeta_{1}(s) \zeta_{2}(s) \\
& \zeta_{P c}(s)=\left(1+2^{-s}-2 \cdot 2^{-2 s}\right) \zeta(s) \zeta_{1}(s) \zeta_{1}(s)+2^{-s} \zeta(s) \zeta_{1}(s) \zeta_{2}(s) \\
& \zeta_{C m}=\left(1+2^{-s}+6 \cdot 2^{-2 s}+8 \cdot 2^{-3 s}\right) \zeta(s) \zeta_{1}(s) \zeta_{1}(s)+2^{-s} \zeta(s) \zeta_{1}(s) \zeta_{2}(s) \\
& \zeta_{C c}(s)=\left(1-3 \cdot 2^{-s}+10 \cdot 2^{-2 s}-8 \cdot 2^{-3 s}\right) \zeta(s) \zeta_{1}(s) \zeta_{1}(s)+2^{-s} \zeta(s) \zeta_{1}(s) \zeta_{2}(s)
\end{aligned}
$$

Theorem 2.2. Normal zeta functions of space groups with the point group isomorphic to the cyclic group of order 2 are given by

$$
\begin{aligned}
& \zeta_{P \overline{1}}^{\triangleleft}(s)=1+14 \cdot 2^{-s}+28 \cdot 2^{-2 s}+8 \cdot 2^{-3 s}+2^{-s} \zeta(s) \zeta_{1}(s) \zeta_{2}(s) \\
& \zeta_{P 2}^{\triangleleft}(s)=\left(1+13 \cdot 2^{-s}+22 \cdot 2^{-2 s}+4 \cdot 2^{-3 s}\right) \zeta(s)+\left(3 \cdot 2^{-2 s}+2^{-s}\right) \zeta(s) \zeta(s) \zeta_{1}(s) \\
& \zeta_{P 21}^{\triangleleft}(s)=\left(1+5 \cdot 2^{-s}-2 \cdot 2^{-2 s}-4 \cdot 2^{-3 s}\right) \zeta(s)+\left(2^{-s}+3 \cdot 2^{-2 s}\right) \zeta(s) \zeta(s) \zeta_{1}(s) \\
& \zeta_{C 2}^{\triangleleft}(s)=\left(2 \cdot 2^{-2 s}+5 \cdot 2^{-s}+1\right) \zeta(s)+2^{-s} \cdot\left(1-2^{-s}+4 \cdot 2^{-2 s}\right) \zeta(s) \zeta(s) \zeta_{1}(s) \\
& \zeta_{P m}^{\triangleleft}(s)=\left(1+11 \cdot 2^{-s}+12 \cdot 2^{-2 s}\right) \zeta(s) \zeta_{1}(s)+2^{-s}\left(1+3 \cdot 2^{-s}\right) \zeta(s) \zeta(s) \zeta_{1}(s) \\
& \zeta_{P c}^{\triangleleft}(s)=\left(1+3 \cdot 2^{-s}-4 \cdot 2^{-2 s}\right) \zeta(s) \zeta_{1}(s)+\left(3 \cdot 2^{-2 s}+2^{-s}\right) \zeta(s) \zeta(s) \zeta_{1}(s) \\
& \zeta_{C m}^{\triangleleft}(s)=\left(3 \cdot 2^{-s}+1\right) \zeta(s) \zeta_{1}(s)+2^{-s} \cdot\left(1-2^{-s}+4 \cdot 2^{-2 s}\right) \zeta(s) \zeta(s) \zeta_{1}(s) \\
& \zeta_{C c}^{\triangleleft}(s)=\left(1-2^{-s}\right) \zeta(s) \zeta_{1}(s)+\left(4 \cdot 2^{-3 s}-2^{-2 s}+2^{-s}\right) \zeta(s) \zeta(s) \zeta_{1}(s)
\end{aligned}
$$

\section{Applications: Subgroups of a given index}

In this and the following section, $d(n)$ denotes the number of all positive divisors of a positive integer $n$ and $\sigma(n)$ denotes the sum of all positive divisors for a positive integer $n$, i. e. $\sigma(n)=\sum_{l \mid n} l$. 
Proposition 3.1. The number of all subgroups of index $n$ in the group $P \overline{1}$ is given by the following expressions

(1) if $n$ is even,

$$
a_{n}=n \sum_{l \mid n} l \cdot \sigma(l)+\sum_{l \mid\left(\frac{n}{2}\right)} l \cdot \sigma(l)
$$

(2) if $n$ is odd, $a_{n}=n \sum_{l \mid n} l \cdot \sigma(l)$,

In particular, $a_{p}=p^{3}+p^{2}+p$ for every odd prime $p$.

Proposition 3.2. The number of all subgroups of index $n$ in group $P 2$ reads:

(1) if $n$ is even,

$$
a_{n}=\sum_{l \mid n} l \cdot \sigma(l)+8 \cdot \sum_{l \mid\left(\frac{n}{2}\right)} l \cdot \sigma(l)
$$

(2) if $n$ is odd, $a_{n}=\sum_{l \mid n} l \cdot \sigma(l)$,

In particular, if $p$ is an odd prime, then $a_{p}=p^{2}+p+1$.

Proposition 3.3. The number of all subgroups of index $n$ in group $P 2_{1}$ is $a_{n}=\sum_{l \mid n} l \cdot \sigma(l)$. In particular, $a_{p}=p^{2}+p+1$ for every odd prime $p$.

Proposition 3.4. The number of all subgroups of index $n$ in the group $C 2$ is

$$
a_{n}=\left\{\begin{array}{l}
\sum_{l \mid n} l \cdot \sigma(l), \quad(n \equiv 1 \vee n \equiv 2 \vee n \equiv 3)(\bmod 4) \\
\sum_{l \mid n} l \cdot \sigma(l)+8 \cdot \sum_{l \mid\left(\frac{n}{4}\right)} l \cdot \sigma(l), n \equiv 0(\bmod 4)
\end{array}\right.
$$

In particular, if $p$ is an odd prime, then $a_{p}=1+p+p^{2}$.

Proposition 3.5. The number of all subgroups of index $n$ in the group Pm is as follows:

(1) if $n$ is even,

$$
a_{n}=\left\{\begin{array}{l}
\sum_{l \mid n} l \cdot d(l)+9 \cdot \sum_{l \mid\left(\frac{n}{2}\right)} l \cdot d(l)+\sum_{l \mid\left(\frac{n}{2}\right)} l \cdot \sigma(l), n \equiv 2(\bmod 4) \\
\sum_{l \mid n} l \cdot d(l)+9 \sum_{l \mid\left(\frac{n}{2}\right)} l \cdot d(l)+6 \sum_{l \mid\left(\frac{n}{4}\right)} l \cdot d(l)+\sum_{l \mid\left(\frac{n}{2}\right)} l \cdot \sigma(l), n \equiv 0(\bmod 4)
\end{array}\right.
$$

(2) if $n$ is odd, $a_{n}=\sum_{l \mid n} l \cdot d(l)$.

In particular, if $p$ is an odd prime, then $a_{p}=2 p+1$.

Proposition 3.6. The number of all subgroups of index $n$ in the group $P c$ is given by: 
(1) if $n$ is even,

$$
a_{n}=\left\{\begin{array}{l}
\sum_{l \mid n} l \cdot d(l)+\sum_{l \mid\left(\frac{n}{2}\right)} l \cdot d(l)+\sum_{l \mid\left(\frac{n}{2}\right)} l \cdot \sigma(l), n \equiv 2(\bmod 4) \\
\sum_{l \mid n} l \cdot d(l)+\sum_{l \mid\left(\frac{n}{2}\right)} l \cdot d(l)-2 \cdot \sum_{l \mid\left(\frac{n}{4}\right)} l \cdot d(l)+\sum_{l \mid\left(\frac{n}{2}\right)} l \cdot \sigma(l), n \equiv 0(\bmod 4)
\end{array}\right.
$$

(2) if $n$ is odd, $a_{n}=\sum_{l \mid n} l \cdot d(l)$.

In particular, if $p$ is an odd prime, then $a_{p}=2 p+1$.

Proposition 3.7. The number of all subgroups of index $n$ in the group $C m$ is:

(1) if $n$ is even,

(2) if $n$ is odd, $a_{n}=\sum_{l \mid n} l \cdot d(l)$.

$$
a_{n}=\left\{\begin{array}{l}
\sum_{l \mid n} l \cdot d(l)+\sum_{l \mid\left(\frac{n}{2}\right)} l \cdot d(l)+\sum_{l \mid\left(\frac{n}{2}\right)} l \cdot \sigma(l),(n \equiv 2 \vee n \equiv 6)(\bmod 8) \\
\sum_{l \mid n} l \cdot d(l)+\sum_{l \mid\left(\frac{n}{2}\right)} l \cdot d(l)+6 \cdot \sum_{l \mid\left(\frac{n}{4}\right)} l \cdot d(l)+\sum_{l \mid\left(\frac{n}{2}\right)} l \cdot \sigma(l), n \equiv 4(\bmod 8) \\
\sum_{l \mid n} l d(l)+\sum_{l \mid\left(\frac{n}{2}\right)} l d(l)+6 \sum_{l \mid\left(\frac{n}{4}\right)} l d(l)+8 \sum_{l \mid\left(\frac{n}{8}\right)} l d(l)+\sum_{l \mid\left(\frac{n}{2}\right)} l \sigma(l), n \equiv 0(\bmod 8)
\end{array}\right.
$$

In particular, if $p$ is an odd prime, then $a_{p}=2 p+1$.

Proposition 3.8. The number of all subgroups of index $n$ in the group $C c$ is the following:

(1) if $n$ is even,

(2) if $n$ is odd, $a_{n}=\sum_{l \mid n} l \cdot d(l)$.

$$
a_{n}=\left\{\begin{array}{l}
\sum_{l \mid n} l \cdot d(l)-3 \cdot \sum_{l \mid\left(\frac{n}{2}\right)} l \cdot d(l)+\sum_{l \mid\left(\frac{n}{2}\right)} l \cdot \sigma(l),(n \equiv 2 \vee n \equiv 6)(\bmod 8) \\
\sum_{l \mid n} l \cdot d(l)-3 \cdot \sum_{l \mid\left(\frac{n}{2}\right)} l \cdot d(l)+10 \cdot \sum_{l \mid\left(\frac{n}{4}\right)} l \cdot d(l)+\sum_{l \mid\left(\frac{n}{2}\right)} l \cdot \sigma(l), n \equiv 4(\bmod 8) \\
\sum_{l \mid n} l d(l)-3 \sum_{l \mid\left(\frac{n}{2}\right)} l d(l)+10 \sum_{l \mid\left(\frac{n}{4}\right)} l d(l)-8 \sum_{l \mid\left(\frac{n}{8}\right)} l d(l)+\sum_{l \mid\left(\frac{n}{2}\right)} l \sigma(l), n \equiv 0(\bmod 8)
\end{array}\right.
$$

In particular, if $p$ is an odd prime, then $a_{p}=2 p+1$.

\section{Applications: Normal subgroups of a given index}

Proposition 4.1. The number of all normal subgroups of index $n$ in the group P $\overline{1}$ reads:

(1) $c_{1}=1$,

(2) if $n$ is odd and $n \neq 1$, then $c_{n}=0$,

(3) if $n$ is even, $c_{n}=\left\{\begin{array}{cc}15, & n=2, \\ 35, & n=4, \\ 43, & n=8, \\ \sum_{l \mid\left(\frac{n}{2}\right)} l \cdot \sigma(l), & n \equiv 0(\bmod 2) \wedge n \neq 1,2,4,8\end{array}\right.$ 
Proposition 4.2. The number of all normal subgroups of index $n$ in the group P2 is:

(2) if $n$ is odd, $c_{n}=1$.

(1) if $n$ is even, $c_{n}=\left\{\begin{array}{l}40+3 \cdot \sum_{l \mid\left(\frac{n}{4}\right)} \sigma(l)+\sum_{l \mid\left(\frac{n}{2}\right)} \sigma(l), n \equiv 0(\bmod 8), \\ 14+\sum_{l \mid\left(\frac{n}{2}\right)} \sigma(l),(n \equiv 2 \vee n \equiv 6)(\bmod 8) \\ 36+3 \cdot \sum_{l \mid\left(\frac{n}{4}\right)} \sigma(l)+\sum_{l \mid\left(\frac{n}{2}\right)} \sigma(l), n \equiv 4(\bmod 8)\end{array}\right.$

Proposition 4.3. The number of all normal subgroups of index $n$ in the group $P 2_{1}$ is given by:

(2) if $n$ is odd, $c_{n}=1$.

$$
3 \cdot \sum_{l \mid\left(\frac{n}{4}\right)} \sigma(l)+\sum_{l \mid\left(\frac{n}{2}\right)} \sigma(l), \quad n \equiv 0(\bmod 8),
$$

(1) if $n$ is even, $c_{n}=\left\{\begin{array}{l}6+\sum_{l \mid\left(\frac{n}{2}\right)} \sigma(l),(n \equiv 2 \vee n \equiv 6)(\bmod 8), \\ 4+3 \cdot \sum_{l \mid\left(\frac{n}{4}\right)} \sigma(l)+\sum_{l \mid\left(\frac{n}{2}\right)} \sigma(l), n \equiv 4(\bmod 8)\end{array}\right.$

Proposition 4.4. The number of all normal subgroups of index $n$ in the group $C 2$ is:

(1) if $n$ is even,

$$
c_{n}=\left\{\begin{array}{l}
6+\sum_{l \mid\left(\frac{n}{2}\right)} \sigma(l),(n \equiv 2 \vee n \equiv 6)(\bmod 8) \\
8-\sum_{l \mid\left(\frac{n}{4}\right)} \sigma(l)+\sum_{l \mid\left(\frac{n}{2}\right)} \sigma(l), n \equiv 4(\bmod 8) \\
8+4 \cdot \sum_{l \mid\left(\frac{n}{8}\right)} \sigma(l)-\sum_{l \mid\left(\frac{n}{4}\right)} \sigma(l)+\sum_{l \mid\left(\frac{n}{2}\right)} \sigma(l), n \equiv 0(\bmod 8)
\end{array}\right.
$$

(2) if $n$ is odd, $c_{n}=1$.

Proposition 4.5. The number of all normal subgroups of index $n$ in the group Pm is:

(1) if $n$ is even,

$$
c_{n}=\left\{\begin{array}{l}
\sigma(n)+11 \cdot \sigma\left(\frac{n}{2}\right)+\sum_{l \mid\left(\frac{n}{2}\right)} \sigma(l), n \equiv 2(\bmod 4) \\
\sigma(n)+11 \cdot \sigma\left(\frac{n}{2}\right)+12 \cdot \sigma\left(\frac{n}{4}\right)+\sum_{l \mid\left(\frac{n}{2}\right)} \sigma(l)+3 \cdot \sum_{l \mid\left(\frac{n}{4}\right)} \sigma(l), n \equiv 0(\bmod 4)
\end{array}\right.
$$

(2) if $n$ is odd, $c_{n}=\sigma(n)$.

In particular, if $p$ is an odd prime, then $c_{p}=p+1$.

Proposition 4.6. The number of all normal subgroups of index $n$ in the group Pc reads: 
(1) if $n$ is even,

$$
c_{n}=\left\{\begin{array}{l}
\sigma(n)+3 \cdot \sigma\left(\frac{n}{2}\right)+\sum_{l \mid\left(\frac{n}{2}\right)} \sigma(l), n \equiv 2(\bmod 4) \\
\sigma(n)+3 \cdot \sigma\left(\frac{n}{2}\right)-4 \cdot \sigma\left(\frac{n}{4}\right)+\sum_{l \mid\left(\frac{n}{2}\right)} \sigma(l)+3 \cdot \sum_{l \mid\left(\frac{n}{4}\right)} \sigma(l), n \equiv 0(\bmod 4)
\end{array}\right.
$$

(2) if $n$ is odd, $c_{n}=\sigma(n)$.

In particular, if $p$ is an odd prime, then $c_{p}=p+1$.

Proposition 4.7. The number of all normal subgroups of index $n$ in the group $C m$ is given by:

(1) if $n$ is even,

$$
c_{n}=\left\{\begin{array}{l}
\sigma(n)+3 \cdot \sigma\left(\frac{n}{2}\right)+\sum_{l \mid\left(\frac{n}{2}\right)} \sigma(l),(n \equiv 2 \vee n \equiv 6)(\bmod 8) \\
\sigma(n)+3 \cdot \sigma\left(\frac{n}{2}\right)+\sum_{l \mid\left(\frac{n}{2}\right)} \sigma(l)-\sum_{l \mid\left(\frac{n}{4}\right)} \sigma(l), n \equiv 4(\bmod 8) \\
\sigma(n)+3 \cdot \sigma\left(\frac{n}{2}\right)+\sum_{l \mid\left(\frac{n}{2}\right)} \sigma(l)-\sum_{l \mid\left(\frac{n}{4}\right)} \sigma(l)+4 \cdot \sum_{l \mid\left(\frac{n}{8}\right)} \sigma(l), n \equiv 0(\bmod 8)
\end{array}\right.
$$

(2) if $n$ is odd, $c_{n}=\sigma(n)$.

In particular, if $p$ is an odd prime, then $c_{p}=p+1$.

Proposition 4.8. The number of all normal subgroups of index $n$ in the group $C c$ is the following:

(1) if $n$ is even,

$$
c_{n}=\left\{\begin{array}{l}
\sigma(n)-\sigma\left(\frac{n}{2}\right)+\sum_{l \mid\left(\frac{n}{2}\right)} \sigma(l),(n \equiv 2 \vee n \equiv 6)(\bmod 8) \\
\sigma(n)-\sigma\left(\frac{n}{2}\right)-\sum_{l \mid\left(\frac{n}{4}\right)} \sigma(l)+\sum_{l \mid\left(\frac{n}{2}\right)} \sigma(l), n \equiv 4(\bmod 8) \\
\sigma(n)-\sigma\left(\frac{n}{2}\right)-\sum_{l \mid\left(\frac{n}{4}\right)} \sigma(l)+\sum_{l \mid\left(\frac{n}{2}\right)} \sigma(l)+4 \cdot \sum_{l \mid\left(\frac{n}{8}\right)} \sigma(l), n \equiv 0(\bmod 8)
\end{array}\right.
$$

(2) if $n$ is odd, $c_{n}=\sigma(n)$.

In particular, if $p$ is an odd prime, then $c_{p}=p+1$.

\section{Proof}

The method for calculating the number of subgroups of any given index in a group $G$ having an abelian normal subgroup $T$ of a finite index (see [6]) could be applied to any polycyclic group or to any poly-(infinite) cyclic-by-finite group. In the sequel, $G$ will denote a space group with the point group isomorphic to the cyclic group of order 2. In each particular case, we make two standard steps. We firstly count all subgroups containing $T$ as its subgroup. Then we count all subgroups contained in $T$. 
In our setting, $G$ is a finite extension of a free abelian group $T$ of rank 3. Group $T$ is generated by three translations $x, y$ and $z$. A subgroup of finite index in $T$ is free and generated by elements $x^{a} y^{b} z^{c}$, $y^{d} z^{e}$ and $z^{f}$. These exponents are unique to the limits: $a, d, f>0,0 \leq b<d, 0 \leq c, e<f$. Since $P$ is a group isomorphic to the cyclic group of order 2 , the index of a subgroup of $T$ in group $G$ is $2 a d f$. We know that the zeta function of $T \cong \mathbb{Z}^{3}$ is $\zeta(s) \zeta(s-1) \zeta(s-2)$. Therefore, the contribution to the zeta function of a group $G$ coming from this part of the problem is $2^{-s} \zeta(s) \zeta(s-1) \zeta(s-2)$ for all eight groups.

Thus, we only need to count subgroups containing $T$. So, let $H_{1}$ be a subgroup containing $T$ as its subgroup. Then $H_{1}$ is generated by elements $r x^{a} y^{b} z^{c}, x^{d} y^{e} z^{f}, y^{g} z^{h}$ and $z^{i}$, where $x, y$ and $z$ are generators of $T$ and $r T$ is a generator of the point group $P$, which is cyclic of order 2 . Furthermore, these exponents are unique to the limits: $d, g, i>0,0 \leq a<d, 0 \leq b, e<g, 0 \leq c, f, h<i$. The index of a subgroup generated by these elements is $d g i$. Since $T$ is a normal subgroup in $G$, then $H_{1} \cap T$ is a normal subgroup in $T$ and

$$
H_{1} /\left(H_{1} \cap T\right) \simeq H_{1} T / T .
$$

This means that $\left(x^{d} y^{e} z^{f}\right)^{r},\left(y^{g} z^{h}\right)^{r}$ and $\left(z^{i}\right)^{r}$ are elements of $H_{1} \cap T$. In this case

$$
H_{1} T / T \simeq P
$$

hence $\left(r x^{a} y^{b} z^{c}\right)^{2}$ is an element of $H_{1} \cap T$. The problem of counting subgroups is reduced to solving the system of equations derived from these conditions. In effect, we consider the number of possible combinations of values (solutions of the corresponding system of equations) which the exponents of the generators of $H_{1}$ may take.

If $H_{1}$ is a normal group in $G$, then its elements have also to satisfy relations $\left(r x^{a} y^{b} z^{c}\right)^{r},\left(r x^{a} y^{b} z^{c}\right)^{x}$, $\left(r x^{a} y^{b} z^{c}\right)^{y},\left(r x^{a} y^{b} z^{c}\right)^{z} \in H_{1}$. Since normality is not a transitive relation, we must also add conditions which will ensure that a normal subgroup of $T$ is a normal subgroup of $G$. Let $H_{2}=<x^{a} y^{b} z^{c}, y^{d} z^{e}, z^{f}>$ be a normal group of $T$. Then $H_{2}$ is a normal subgroup of $G$, if $\left(x^{a} y^{b} z^{c}\right)^{r},\left(y^{d} z^{e}\right)^{r}$ and $\left(z^{f}\right)^{r}$ are elements of $\mathrm{H}_{2}$.

When writing a space group in an abstract form, we follow the descriptions of these groups given in [5]. The software packages Mathematica Wolfram and GAP were apt for double checking the calculations. Mathematica was used to convert our formulas into lists of integers $a_{n}$ or $c_{n}$.

We demonstrate the above technique in detail in the case of group $C 2$.

\subsection{Zeta function of group $C 2$.}

Recall that $C 2=\left\langle x, y, z, r \mid[x, y],[x, z],[y, z], r^{2}, x^{r}=x y, y^{r}=y^{-1}, z^{r}=z^{-1}\right\rangle$.

We are counting subgroups of the form $H_{1}=\left\langle r x^{a} y^{b} z^{c}, x^{d} y^{e} z^{f}, y^{g} z^{h}, z^{i}\right\rangle$. Each of $\left(r x^{a} y^{b} z^{c}\right)^{2}$, $\left(x^{d} y^{e} z^{f}\right)^{r},\left(y^{g} z^{h}\right)^{r},\left(z^{i}\right)^{r}$ must lie in $H_{1} \cap T$. Now, $\left(r x^{a} y^{b} z^{c}\right)^{2}=x^{2 a} y^{a},\left(x^{d} y^{e} z^{f}\right)^{r}=x^{-d} y^{-e} z^{-f}$. $x^{2 d} y^{d},\left(y^{g} z^{h}\right)^{r}=y^{-g} z^{-h},\left(z^{i}\right)^{r}=z^{-i}$. Each of $x^{-d} y^{-e} z^{-f}, y^{-g} z^{-h}, z^{-i}$ is contained in $H_{1} \cap T=$ $\left\langle x^{d} y^{e} z^{f}, y^{g} z^{h}, z^{i}\right\rangle$, regardless of the values of $d, e, f, g, h, i$. So, $\left(r x^{a} y^{b} z^{c}\right)^{2}$ lies in $H_{1} \cap T$ if $x^{2 a} y^{a}$ lies 
in $H_{1} \cap T ;\left(x^{d} y^{e} z^{f}\right)^{r}$ lies in $H_{1} \cap T$ if $x^{2 d} y^{d}$ lies in $H_{1} \cap T$. If $x^{2 a} y^{a}, x^{2 d} y^{d}$ are in $H_{1} \cap T$ then there exist integers $\alpha_{1}, \alpha_{2}, \beta_{1}, \beta_{2}, \gamma_{1}, \gamma_{2}$ such that:

$x^{2 d} y^{d}=\left(x^{d} y^{e} z^{f}\right)^{\alpha_{1}}\left(y^{g} z^{h}\right)^{\beta_{1}}\left(z^{i}\right)^{\gamma_{1}}, x^{2 a} y^{a}=\left(x^{d} y^{e} z^{f}\right)^{\alpha_{2}}\left(y^{g} z^{h}\right)^{\beta_{2}}\left(z^{i}\right)^{\gamma_{2}}$.

We get the following system of equations:

$$
S_{1}=\left\{\begin{array}{l}
d \alpha_{1}=2 d, e \alpha_{1}+g \beta_{1}=d, f \alpha_{1}+h \beta_{1}+i \gamma_{1}=0, \\
d \alpha_{2}=2 a, e \alpha_{2}+g \beta_{2}=a, f \alpha_{2}+h \beta_{2}+i \gamma_{2}=0,
\end{array}\right\} .
$$

The first equation implies $\alpha_{1}=2$. Consider the equation $2 e=-g \beta_{1}+d$. The left side of the equation is even. If $g$ is even, then $d$ has also to be even. We get: $\frac{d}{g} \geq \beta_{1}>-2+\frac{d}{g}$. There are two integers in the interval $\left[\frac{d}{g}, \frac{d}{g}-2\right)$. To solve the above system of equations, we shall consider the following cases:

Case 1. $d, g, i$ are odd. In this case, there is one choice for $a$. Since $\beta_{1}$ has to be odd, there is one choice for $e$. From $2 f+h \beta_{1}+i \gamma_{1}=0$, we get $-\frac{h \beta_{1}}{i} \geq \gamma_{1}>-2-\frac{h \beta_{1}}{i}$. Now, $h \beta_{1}$ and $i \gamma_{1}$ have to be odd or even at the same time. Since $\beta_{1}$ and $i$ are odd, we conclude that if $h$ is odd then $\gamma_{1}$ is odd and if $h$ is even then $\gamma_{1}$ is even. Hence, there are $i$ choices for $h$ and one choice for $f$. The zeta function contribution in this case is: $\sum_{d, g, i \in \mathbb{N}^{\prime}} d^{-s} g^{-s} i^{-s} g i i$, where $\mathbb{N}^{\prime}=\{2 k-1 \mid k \in \mathbb{N}\}$.

Case 2. and Case 3. ( $d, i$ are odd, $g$ is even) and ( $d$ is odd, $g, i$ are even). These cases are impossible.

Case 4. $i, g$ are odd, $d$ is even. There are two choices for $a$. Since $\beta_{1}$ has to be even, there is one choice for $e$. Furthermore, $\gamma_{1}$ has to be even, so there is one choice for $f$. The zeta function contribution in this case is: $2 \cdot \sum_{d \in 2 \mathbb{N}, g, i \in \mathbb{N}^{\prime}} d^{-s} g^{-s} i^{-s}$ gii, where $\mathbb{N}^{\prime}=\{2 k-1 \mid k \in \mathbb{N}\}$.

Case 5. $g, d$ are odd, $i$ is even. There is one choice for $a ; \beta_{1}$ has to be odd, so there is one choice for $e$. Since $h \beta_{1}$ and $i \gamma_{1}$ have to be odd or even at the same time, we see that $h$ has to be even. There are two choices for $\gamma_{1}$, hence there are two choices for $f$. The zeta function contribution in this case is: $\sum_{i \in 2 \mathbb{N}, g, d \in \mathbb{N}^{\prime}} d^{-s} g^{-s} i^{-s} g i \cdot \frac{i}{2} \cdot 2$.

Case 6. $g$ is odd, $i, d$ are even. There are two choices for $a ; \beta_{1}$ has to be even, so there is one choice for $e$. If $a=0$, then there are two choices for $f$; if $a=\frac{d}{2}$ then $\gamma_{1}$ is even, so there is one choice for $f$ in this case. The zeta function contribution reads: $3 \sum_{g \in \mathbb{N}^{\prime}, d, i \in 2 \mathbb{N}} d^{-s} g^{-s} i^{-s} g i i$.

Case 7. $g, d$ are even, $i$ is odd. There are two choices for $a$. If $a=0$ and $\beta_{1}$ is even, then $\gamma_{1}$ is even and there are $i$ choices for $h$. Hence, for $a=0$ and $\beta_{1}$ is even, there are one choice for $e$ and one choice for $f$. If $a=0$ and $\beta_{1}$ is odd, then $\gamma_{1}$ and $h$ are both even or both odd. If $a=\frac{d}{2}$ then $\beta_{1}, \gamma_{1}$ are even and there is one choice for $f$. The zeta function contribution in this case is: $3 \sum_{i \in \mathbb{N}^{\prime}, d, g \in 2 \mathbb{N}} d^{-s} g^{-s} i^{-s} g i i$.

Case 8. $g, d i$ are even. There are two choices for $a$. If $a=0$ and $\beta_{1}$ is even, then there are two choice for $\gamma_{1}$ and there are $i$ choices for $h$. If $a=0$ and $\beta_{1}$ is odd, then there are two choices for $\gamma_{1}$ and $h$ is even $\left(\frac{i}{2}\right.$ choices for $h$ ). If $a=\frac{d}{2}$ then $\beta_{1}, \gamma_{1}$ are even and there is one choice for $f$. The zeta 
function contribution in this case is:

$\sum_{d, i, g \in 2 \mathbb{N}} d^{-s} g^{-s} i^{-s} \cdot g \cdot i \cdot i \cdot 2+\sum_{d, i, g \in 2 \mathbb{N}} d^{-s} g^{-s} i^{-s} \cdot g \cdot i \cdot \frac{i}{2} \cdot 2+\sum_{d, i, g \in 2 \mathbb{N}} d^{-s} g^{-s} i^{-s} \cdot g \cdot i \cdot i=4 \sum_{d, i, g \in 2 \mathbb{N}} d^{-s} g^{-s} i^{-s} \cdot g \cdot i \cdot i$.

Finally, we obtain the zeta function for group $C 2$ :

$$
\begin{aligned}
& \zeta_{C 2}(s)=\sum_{d, g, i \in \mathbb{N}^{\prime}} d^{-s} g^{-s} i^{-s} g i i+2 \cdot \sum_{d \in 2 \mathbb{N}, g, i \in \mathbb{N}^{\prime}} d^{-s} g^{-s} i^{-s} g i i+ \\
+ & \sum_{d, g \in \mathbb{N}^{\prime}, i \in 2 \mathbb{N}} d^{-s} g^{-s} i^{-s} g i i+3 \sum_{g \in \mathbb{N}^{\prime}, i, d \in 2 \mathbb{N}} d^{-s} g^{-s} i^{-s} g i i+3 \sum_{i \in \mathbb{N}^{\prime}, d, g \in 2 \mathbb{N}} d^{-s} g^{-s} i^{-s} g i i+ \\
+ & 4 \sum_{d, i, g \in 2 \mathbb{N}} d^{-s} g^{-s} i^{-s} g i i+2^{-s} \zeta(s) \zeta(s-1) \zeta(s-2)=\left(1+2^{-2 s+3}\right) \zeta(s) \zeta_{1}(s) \zeta_{2}(s) .
\end{aligned}
$$

\subsection{Normal zeta function of group $C 2$.}

We use the set of constraints which we obtained in the previous Subsection. By counting the number of subgroups $H_{1}=\left\langle r x^{a} y^{b} z^{c}, x^{d} y^{e} z^{f}, y^{g} z^{h}, z^{i}\right\rangle$ of $C_{2}$, we deduced the system $S_{1}$.

Based on the conditions of normality, we get another set of constraints:

$$
S_{2}=\left\{\begin{array}{l}
d \alpha_{3}=0, e \alpha_{3}+g \beta_{3}=-1, f \alpha_{3}+h \beta_{3}+i \gamma_{3}=0 \\
d \alpha_{4}=0, e \alpha_{4}+g \beta_{4}=2, f \alpha_{4}+h \beta_{4}+i \gamma_{4}=0 \\
d \alpha_{5}=0, e \alpha_{5}+g \beta_{5}=0, f \alpha_{5}+h \beta_{5}+i \gamma_{5}=2
\end{array}\right\}
$$

The equations $d \alpha_{3}=0, e \alpha_{3}+g \beta_{3}=-1, f \alpha_{3}+h \beta_{3}+i \gamma_{3}=0$ imply that $g=1, \beta_{3}=-1$, so $b=e=0=h$, while the equations $d \alpha_{5}=0, e \alpha_{5}+g \beta_{5}=0, f \alpha_{5}+h \beta_{5}+i \gamma_{5}=2$ imply that $i=1$ or $i=2$. Observing four cases depending on whether $d$ is even or odd and depending on values of $i$, we get

$\sum_{d \in \mathbb{N}^{\prime}} d^{-s}+2 \sum_{d \in 2 \mathbb{N}} d^{-s}+4 \cdot 2^{-s} \sum_{d \in \mathbb{N}} d^{-s}+6 \sum_{d \in 2 \mathbb{N}} d^{-s} 2^{-s}=\left(2 \cdot 2^{-2 s}+5 \cdot 2^{-s}+1\right) \zeta(s)$.

Now, we count normal subgroups of $T$. Any such subgroup takes the form $H_{2}=\left\langle x^{a} y^{b} z^{c}, y^{d} z^{e}, z^{f}\right\rangle$. We assume $0<a, 0 \leq b<d, 0 \leq c, e<f$. Let us check the conditions of normality in $C 2$. These require that $\left(x^{a} y^{b} z^{c}\right)^{r},\left(y^{d} z^{e}\right)^{r},\left(z^{f}\right)^{r}$ are in $H_{2}$. After some calculations, we get the next set of constraints:

$$
S_{3}=\left\{a \alpha_{1}=2 a, b \alpha_{1}+d \beta_{1}=a, c \alpha_{1}+e \beta_{1}+f \gamma_{1}=0\right\}
$$

This system of equations reduces to three equations: $d \beta_{1}=a-2 b, e \beta_{1}+f \gamma_{1}=0,-2 e+f \gamma_{1}=0$. The case $a$ odd and $d$ even is impossible. Thus, we have six cases depending on whether $a$ or $d$ or $f$ are odd or even. The respective contributions are:

$$
\begin{array}{r}
\sum_{a, d, f \in \mathbb{N}^{\prime}} a^{-s} d^{-s} f^{-s} f+\sum_{a \in 2 \mathbb{N}, d, f \in \mathbb{N}^{\prime}} a^{-s} d^{-s} f^{-s} f+2 \sum_{a, f \in 2 \mathbb{N}, d \in \mathbb{N}^{\prime}} a^{-s} d^{-s} f^{-s} f+ \\
+2 \sum_{a, d \in 2 \mathbb{N}, f \in \mathbb{N}^{\prime}} a^{-s} d^{-s} f^{-s} f+\sum_{a, d \in \mathbb{N}^{\prime}, f \in 2 \mathbb{N}} a^{-s} d^{-s} f^{-s} f+3 \sum_{a, d, f \in 2 \mathbb{N}} a^{-s} d^{-s} f^{-s} f
\end{array}
$$


$=\left(1-2^{-s}+4 \cdot 2^{-2 s}\right) \zeta(s) \zeta(s) \zeta(s-1)$.

Combining the contributions coming from $H_{1}$ and $H_{2}$, we get the normal zeta function of $C 2$ :

$\zeta_{C 2}^{\triangleleft}(s)=\left(2 \cdot 2^{-2 s}+5 \cdot 2^{-s}+1\right) \zeta(s)+2^{-s} \cdot\left(1-2^{-s}+4 \cdot 2^{-2 s}\right) \zeta(s) \zeta(s) \zeta_{1}(s)$.

\section{Remarks}

As far as subsection 5.1 is concerned, using a similar argumentation one can obtain more general assertions of the following form.

Proposition 6.1. Let $n \geq 2$ be a fixed integer and $G_{n}$ be the group defined by

$G_{n}=\left\langle r, x_{1}, x_{2}, \ldots, x_{n} \mid r^{2},\left[x_{j}, x_{k}\right](\forall 1 \leq j, k \leq n), x_{i}{ }^{r}=x_{i}{ }^{-1}(\forall 1 \leq i \leq n)\right\rangle$. The zeta function of group $G_{n}$ is given by

$$
\zeta_{G_{n}}(s)=\zeta(s-1) \zeta(s-2) \cdots \zeta(s-n)+2^{-s} \zeta(s) \zeta(s-1) \zeta(s-2) \cdots \zeta(s-n+1) .
$$

Proposition 6.2. Let $n \geq 2$ be an integer and $G_{n}$ be the group defined by

$G_{n}=\left\langle r, x_{1}, x_{2}, \ldots, x_{n} \mid r^{2},\left[x_{j}, x_{k}\right](\forall 1 \leq j, k \leq n), x_{1}{ }^{r}=x_{1} x_{2}, x_{i}{ }^{r}=x_{i}{ }^{-1}(\forall 1<i \leq n)\right\rangle$. The zeta function of group $G_{n}$ is $\zeta_{G_{n}}(s)=\left(1+2^{-2 s+n}\right) \zeta(s) \zeta(s-1) \zeta(s-2) \cdots \zeta(s-n+1)$. The zeta function of group $G_{n}$ has an Euler product.

\section{REFERENCES}

[1] du Sautoy MPF, Lubotzky A., Functional equations and uniformity for local zeta functions of nilpotent groups., Amer J Math. 118 no. 4 (1996), 39-90.

[2] du Sautoy MPF, McDermott JJ, Smith GC, Zeta functions of crystallographic groups and analytic continuation Proc Lond Math Soc. 79 ( 2010), 511-534.

[3] du Sautoy MPF, Woodward L. Zeta functions of groups and rings, Lecture notes in mathematics 1925, SpringerVerlag, 2008.

[4] Grunewald FJ, Segal D, Smith GC. Subgroups of finite index in nilpotent groups.Invent Math. 93 (1988), $185-223$.

[5] Lord E. Generators and relations for space groups. Bangalore, 2010.

[6] McDermott JJ. Subgroup growth and the plane crystallographic groups. PhD, University of Bath, UK, 1997.

[7] Smith GC. Zeta-functions of torsion-free finitely generated nilpotent groups. PhD, University of Manchester, UK, 1983.

[8] C. Voll, Functional equations for zeta functions of groups and rings. Annals of Mathematics 172 (2010), 1181-1218.

[9] C. Voll, A. Beauville, Functional equations for local normal zeta functions of nilpotent groups. Geometric and Functional Analysis GAFA 15 (2005), 274-295. 